\title{
Validation of full-field techniques: discussion of experiences
}

\author{
E. Hack ${ }^{1, \mathrm{a}}$, R. Burguete ${ }^{2}$, T. Siebert ${ }^{3}$, A. Davighi ${ }^{4}$ J. Mottershead $^{5}$, G. Lampeas ${ }^{6}$, A. Ihle ${ }^{7}$, \\ E.A. Patterson ${ }^{8}$, and A. Pipino ${ }^{9}$ \\ ${ }^{1}$ Empa, Laboratory Electronics/Metrology/Reliability, CH-8600 Dübendorf, Switzerland \\ ${ }^{2}$ Airbus UK Ltd, New Filton House, Filton, Bristol, BS99 7AR, United Kingdom \\ ${ }^{3}$ Dantec Dynamics GmbH, Kässbohrerstr. 18, D-89077 Ulm, Germany \\ ${ }^{4}$ EC Joint Research Centre - IHCP, Via Enrico Fermi 1, I-21020 Ispra (VA), Italy \\ ${ }^{5}$ University of Liverpool, Department of Engineering, Liverpool, L69 7ZF, United Kingdom \\ ${ }^{6}$ University of Patras, Rion Patra, HE-26504 Patras, Greece \\ ${ }^{7}$ High Performance Space Structure Systems GmbH, D-38112, Braunschweig, Germany \\ ${ }^{8}$ Michigan State University, Department of Mechanical Engineering, East Lansing, MI 48824, USA \\ ${ }^{9}$ Centro Ricerche Fiat S.C.p.A., Strada Torino 50, I-10043 Orbassano, Italy
}

\begin{abstract}
Validation and calibration of optical full-field techniques that are used to measure strain and displacement in experimental mechanics is a prerequisite for validating numerical stress analyses. ICEM14 brings together practising engineers from around the world to exchange their experience regarding validation and calibration from everyday measurements with different optical techniques. The discussion addresses the following issues: (i) experience in calibrating measurement equipment based on imaging; (ii) reference measurements and calibration artefacts; (iii) validation of finite element analyses by comparison to experimental data; and (iv) uncertainties in full-field measurements.
\end{abstract}

\section{Introduction}

Full-field optical techniques are routinely used to measure strain and displacement in experimental mechanics. Calibration and validation of these techniques should be a necessary task, despite the fact that many experimenters do not quantify the accuracy or measurement uncertainty of their results. In ICEM14 session B10, Validation and Standards for Dynamic Analyses, these topics will be addressed, discussion invited, and some response received [1-7]. The topic will be placed in the context of projects at the European and global levels [8,9]. This International Conference on Experimental Mechanics brings together practising engineers from around the world and provides an opportunity to exchange their experience regarding the calibration of their full-field measurement instruments, the use of reference artefacts for everyday measurements, the expression of measurement uncertainty, as well as the use of imaging measurement equipment for the validation of numerical modelling results.

Therefore, we hope for an open discussion and exchange of views and experiences from the audience and the authors in the session that will provide stimulus and direction for future

\footnotetext{
${ }^{\mathrm{a}}$ e-mail: erwin.hack@empa.ch
} 
development of protocols for calibration and validation. This paper provides a framework of the discussion around the following issues:

- Experience in calibrating imaging measurement equipment

- Reference measurements and calibration artefacts

- Full-field measurement uncertainty

- Validation of finite element (FE) analysis results by comparison to experimental data

\section{Calibrating imaging measurement equipment}

Today, the use of whole field optical techniques is ubiquitous. Deformations, strain, or vibration modes due to defined loads are often measured by digital image correlation methods (DIC) or fringe techniques such as moiré, holographic and digital speckle pattern interferometry (DSPI) or shearography. For dynamic events, the DIC method can easily be applied using high speed cameras and delivers quantitative information on $3 \mathrm{D}$ displacements.

Calibration of displacement and strain measurement systems is an essential step in providing traceability to a national standard and confidence in stress and strain distributions obtained from experiment and used to validate simulations employed in engineering design. Calibration also provides data on the uncertainty of measurements obtained with the calibrated measurement system which can be used to define confidence limits for experimental data. How can a full-field technique be calibrated? And how should the measurement uncertainty be expressed in this case? Obviously, a field of reference values must be provided to calibrate a full-field technique. The need arises for an artefact that allows a full-field calibration of the quantity in question. The requirements for such an artefact from the point of view of traceability of displacement or strain measurement, its realization for planar, static strain states and its use for calibrating an optical instrument has been described. The Standardisation Project for Optical Techniques of Strain measurement (SPOTS) developed and published a reference material and procedure for the calibration of optical systems of full-field strain measurement. As an exemplar for the calibration process the calibration of a commercially available DIC system for a single component of the normal in-plane strain has been presented in this session and previously for an electronic speckle pattern interferometer [10].

In order to calibrate instruments for dynamic measurements of displacement or strain the limitations of a static calibration must be overcome. The motivation for this extension is the use of optical measurement systems for capturing dynamic events such as vibrations or impact. In the $7^{\text {th }}$ Framework Programme of the European Union the ADVISE project was started to tackle this challenge [09]. The objectives of the ADVISE project are:

- development of reference materials that allow traceability and calibration of full-field optical methods of deformation measurement in cyclic, transient and non-linear dynamic events;

- optimisation of methodologies for both optical measurement and computational modelling and simulation of non-linear, transient dynamic events;

- contributions to standardisation activity for experimental validation of dynamic simulations.

\section{Reference measurements and calibration artefacts}

Reference materials provide a simple, well-defined distribution of the measured quantity that can be traced to an international standard and can be used to assess the uncertainty associated with the measurement system. Previous work has established a reference material and procedure for 
calibrating optical systems for measuring static, in-plane strain distributions and also demonstrated its use. The reference material consists of a beam in four-point bending within a monolithic frame that ensures reproducibility of the boundary and loading conditions. A procedure for the use of the reference material has been developed and leads to the evaluation of measurement uncertainties that allow the construction of confidence limits for the data obtained with the calibrated optical system. The design and methodology have been developed into a proposed draft standard which is being endorsed by VAMAS (Versailles Agreement on Materials And Standards) for review by ISO (International Organisation for Standardization).

A new effort is in progress to extend this work to the measurement of three-dimensional displacement and strain distributions induced by cyclic and dynamic loading, including transients and large-scale deformation. The first step in this effort has been to define both the essential and desirable attributes of a reference material for calibrating systems capable of measurements of dynamic displacement and strain. The attributes have been utilised to evaluate designs for the reference material. The final selection and embodiment of the design for the reference material has been presented together with the test program for its evaluation.

\section{Full-field measurement uncertainty}

When calibrating an instrument such as an LVDT, a single reference quantity is captured by the transducer using a sequence of well-defined reference values. The reading of the transducer is compared to the reference value, and the residual deviations from a linear function are quantified. Usually a single uncertainty value (or specification limit) is extracted from the deviations.

An array detector can be seen as a huge number of transducers working in parallel. Therefore, the calibration field has to be provided simultaneously and not sequentially. Although the Guide to the Expression of Uncertainty in Measurement (GUM [11]) contains detailed information on how to obtain the uncertainty from a model description of the measurement process, there is no description of a procedure to tackle full-field data acquisition and processing.

\section{Validation of FE analyses by comparison to experimental data}

In engineering modelling the analysis of homogeneous materials has become a fairly routine process. The most common approaches to such design involve simulations based on Finite Element Analysis (FEA), used to study the mechanical response of the structural elements during critical events. Recent advances in material technology allow the use of light-weight yet impact-resistant fibre reinforced polymers. Since impact testing is a cost intensive venture, the replacement of actual experimental testing by reliable, validated simulations is a goal of many designers. Impact analysis methodologies have advanced significantly in recent years, using a variety of approaches, and various commercial FE codes are available that provide sophisticated capabilities. However, currently there is no universally accepted impact simulation methodology, and too many numerical parameters require an experimental determination.

It is good practice to validate analytical and numerical models used in stress analysis by comparison with measurements obtained from real components. Modern techniques of optical analysis allow full-field maps of displacement, strain or stress to be obtained from real components with relative ease. However, validations continued to be performed only at predicted and, or observed hot-spots and most of the wealth of experimental data is ignored. In addition to point-by-point comparisons of e.g. experimental and calculated load-deflection curves, it has been proposed to employ image decomposition methods to validate stress analysis models [7]. Image decomposition techniques such as Zernike moments and Fourier transforms have been used to decompose full-field distributions for 
strain generated from optical techniques such as digital image correlation and thermoelastic stress analysis as well as from analytical and numerical models by treating the strain distributions as images. As a consequence, it is relatively easy to make a statistical comparison of the image descriptors from the experiment and from the analytical/numerical model and to provide a quantitative assessment of the stress analysis. The technique can also be used to perform the chain of comparisons that are necessary to generate traceability for measurements to a national standard. Examples of the application of the proposed method to composite panels have been presented and discussed.

\section{Acknowledgement}

Part of this work has received funding from the European Community's Seventh Framework Programme under Grant Agreement no. SCP7-GA-2008-218595 (ADVISE project).

\section{References}

1. Hack, E., Patterson, E., Siebert, T., and Thalmann, R., Calibration and validation of full-field techniques, ICEM 14, Poitiers (FR), 4 - 9 July 2010, paper 491

2. Burguete, R.L., and Patterson, E.A., SPOTS project and development of standard and reference material, ICEM 14, Poitiers (FR), 4 - 9 July 2010, paper 445

3. Sebastian, C., Patterson, E.A., SPOTS calibration example, ICEM 14, Poitiers (FR), 4 - 9 July 2010, paper 460

4. Davighi, A., Patterson, E.A., Whelan, M., A reference material for calibration of dynamic displacement and strain measurements, ICEM 14, Poitiers (FR), 4 - 9 July 2010, paper 305

5. Feligiotti, M., Hack, E., and Lampeas, G., Methodology for assessing impact damage using integrated simulation and experimentation, ICEM 14, Poitiers (FR), 4 - 9 July 2010, paper 332

6. Lampeas, G., and Siebert, T., Validation of non-linear dynamic simulations through full field optical methods, ICEM 14, Poitiers (FR), 4 - 9 July 2010, paper 321

7. Patki, A., Wang, V., Mottershead, J., Patterson, E.A., Image decomposition as a tool for validating stress analysis models, ICEM 14, Poitiers (FR), 4 - 9 July 2010, paper 271

8. SPOTS: Standardisation project for optical techniques of strain measurement, EU contract no. G6RD-CT-2002-00856, see http://www.opticalstrain.org.

9. ADVISE: Advanced Dynamic Validations using Integrated Simulation and Experimentation, FP7 project SCP7-GA-2008-218595, see http://www.dynamicvalidation.org.

10. Whelan, M.P., Albrecht, D., Hack, E., Patterson, E.A., 2008, 'Calibration of a speckle interferometry full-field strain measurement system', Strain, 44(2):180-190.

11. GUM Guide to the expression of uncertainty in measurement, Joint Committee for Guides in Metrology 100:2008 (BIPM, Paris, 2008). 